\title{
Complements of nearly perfect graphs
}

\author{
András Gyárfás*, Zhentao Li ${ }^{\dagger}$, Raphael Machado ${ }^{\ddagger}$, \\ András Sebő ${ }^{\S}$, Stéphan Thomassé $e^{\S}$ And Nicolas Trotignon ${ }^{\S}$
}

\begin{abstract}
A class of graphs closed under taking induced subgraphs is $\chi$ bounded if there exists a function $f$ such that for all graphs $G$ in the class, $\chi(G) \leq f(\omega(G))$. We consider the following question initially studied in [A. Gyárfás, Problems from the world surrounding perfect graphs, Zastowania Matematyki Applicationes Mathematicae, 19:413-441, 1987]. For a $\chi$-bounded class $\mathcal{C}$, is the class $\bar{C} \chi$-bounded (where $\overline{\mathcal{C}}$ is the class of graphs formed by the complements of graphs from $\mathcal{C})$ ?

We show that if $\mathcal{C}$ is $\chi$-bounded by the constant function $f(x)=$ 3 , then $\overline{\mathcal{C}}$ is $\chi$-bounded by $g(x)=\left\lfloor\frac{8}{5} x\right\rfloor$ and this is best possible. We show that for every constant $c>0$, if $\mathcal{C}$ is $\chi$-bounded by a function $f$ such that $f(x)=x$ for $x \geq c$, then $\overline{\mathcal{C}}$ is $\chi$-bounded. For every $j$, we construct a class of graphs $\chi$-bounded by $f(x)=x+x / \log ^{j}(x)$ whose complement is not $\chi$-bounded.
\end{abstract}

\section{Introduction}

In the present paper, we consider simple and finite graphs. We denote by $\chi(G)$ (resp. $\theta(G)$ ) the minimum number of stable sets (resp. cliques) needed to cover the vertices of $G$. We denote by $\omega(G)$ (resp. $\alpha(G)$ ) the maximum size of a clique (resp. stable set) of $G$. A graph $G$ is $\chi$-bounded (resp. $\theta$-bounded) by a function $f$ if $\chi(H) \leq f(\omega(H)$ ) (resp. $\theta(H) \leq f(\alpha(H))$ ) for every induced subgraph $H$ of $G$. Observe that a graph $G$ is $\chi$-bounded by $f$ if an only if its complement $\bar{G}$ is $\theta$-bounded by $f$. A class of graphs is $\chi$-bounded (resp. $\theta$-bounded) if for some function $f$, every graph of the class is $\chi$-bounded (resp. $\theta$-bounded) by $f$. The class of perfect graphs is the class of graph

arXiv: 1304.2862

*Research supported in part by OTKA Grant No. K104343.

${ }^{\dagger}$ Research partially supported by the FQRNT.

${ }^{\ddagger}$ Partially supported by CNPq and Faperj.

${ }^{\S}$ Partially supported by the French Agence Nationale de la Recherche under reference ANR-10-BLAN 0207TEOMATRO, ANR-10-JCJC-HEREDIA and ANR-14-BLAN STINT. 
$\chi$-bounded by the identity function. For every $\chi$-bounded (resp. $\theta$-bounded) class, there exists a smallest $\chi$-bounding (resp. $\theta$-bounding) function that we refer to as the optimal $\chi$-bounding (resp. $\theta$-bounding) function for the class.

We address a general question asked by Gyárfás [4]: for which functions $f$ is the class of graphs $\chi$-bounded by $f$ also $\theta$-bounded (by a possibly different function $g$ )? Such functions are called complementary-bounded functions and $g$ is a complementary bounding function for $f$. If $f$ is a complementary bounding function, we denote by $f^{*}$ the optimal $\theta$-bounding function of the class of graphs $\chi$-bounded by $f$.

Theorem 1.1 (König [9]). If $G$ is bipartite, then $\theta(G)=\alpha(G)$.

The classical theorem above can be rephrased as "the identity is the optimal complementary bounding function for the constant function $f=2$ ", or by "2* = id". In Section 2, we push further this line of research by computing the optimal complementary bounding function of the constant function $f=3$. To do so, we prove that 3 -colourable graphs are $\theta$-bounded by $f^{*}(x)=\left\lfloor\frac{8}{5} x\right\rfloor$ or equivalently $3^{*}=\left\lfloor\frac{8}{5} \mathrm{id}\right\rfloor$ (Theorem 2.1). Our proof uses a well-known result of Gallai [3] on color critical graphs (Theorem 2.3 below).

The following remarks on the constant function $f_{m}=m$ are from [4]. On one hand, $f_{m}^{*}(x) \leq\left\lfloor\frac{m+1}{2}\right\rfloor x$ because the vertex set of any $m$-chromatic graph can be covered by the vertices of at most $\left\lfloor\frac{m+1}{2}\right\rfloor$ bipartite graphs. On the other hand, $f_{m}^{*}(x) \geq \frac{m x}{2}$ for $x>x_{0}(m)$ from a nice probabilistic construction of Erdős [1]: for arbitrary $m$, there exists $t$ and an $m$-partite triangle-free graph $G$ with $t$ vertices in each partite class, such that $\alpha(G)=t$. In fact, our construction on Fig. 1 showing $f_{3}^{*}(x) \geq\left\lfloor\frac{8}{5} x\right\rfloor$ is such a graph with $m=3, t=5$. Thus, we have the asymptotic of $f_{m}^{*}$ for even $m$ 's, but not for odd $m$ 's, apart from $m=3$. It seems hard to make an intelligent guess even on the asymptotic $f_{5}^{*}(x)$.

Kőnig's theorem was generalized by Lovász in the Perfect Graph Theorem [10], stating that the identity is its own optimal complementary bounding function. A function $f$ is eventually identity if there exist a constant $c$ such that for all $x \geq c, f(x)=x$. In Section 3, we prove eventually identity functions are complementary-bounded (Theorem 3.1). This theorem was stated without proof in [4]. Our proof is an induction that reduces the problem to Lovász's theorem.

In Section 4, we deal with functions that are not complementary-bounded. Gyárfás [4] proved that for every real number $\varepsilon>0$, the function $f(x)=$ $x+\varepsilon x$ (and thus any function greater than $f$ ) is not complementary bounded. We improve this result by proving that the function $f(x)=x+x / \log ^{j}(x)$ is 

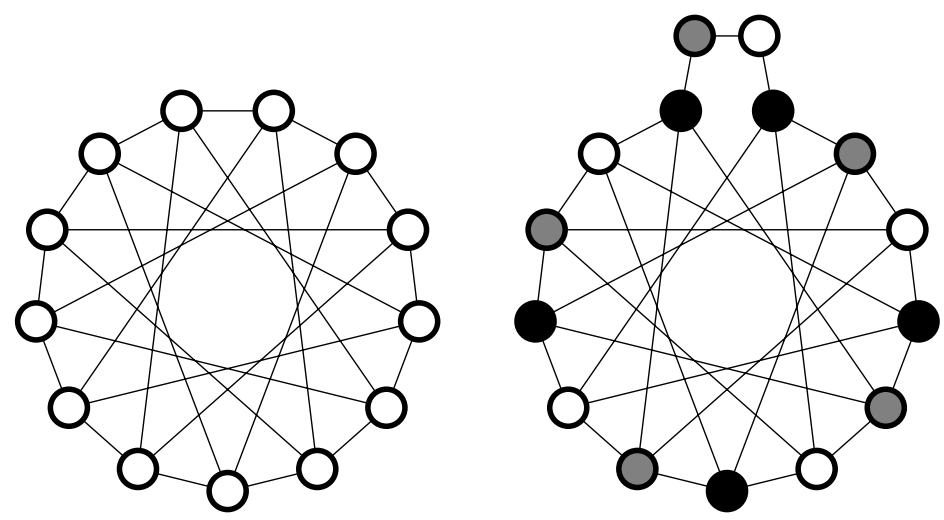

Figure 1: Graphs $R_{3,5}$ and $G_{5,8}$.

not-complementary-bounded for any $j$ (Theorem 4.8). The methods we use to prove $\chi$-boundedness in this section rely again on a theorem of Gallai [2] on factor-critical graphs, graphs where removing any vertex yields a graph with a perfect matching.

It was conjectured in [4] that $f(x)=x+c$ is complementary-bounded for any constant $c$. This conjecture remains open even for $c=1$. Our tools from Sections 2 and 3 are not strong enough to prove that $f$ is complementarybounded, and our tools from Section 4 are not strong enough to prove that it is not.

\section{Complementary bounding function of 3-chromatic graphs}

In this section, we find the smallest $\theta$-bounding function for the class of 3 -colourable graphs. We work on a more general class $\mathcal{C}$ : graphs $G$ such that for every induced subgraph $H$ of $G, \alpha(H) \geq|V(H)| / 3$. Graphs satisfying this property are more general than 3 -colourable graphs as the 5 -wheel has this property but is not 3-colourable.

Theorem 2.1. Every graph $G$ in $\mathcal{C}$ satisfies $\theta(G) \leq\left\lfloor\frac{8}{5} \alpha(G)\right\rfloor$. This is best possible, in the sense that for every integer $x \geq 0$, there exists a graph $G$ in $\mathcal{C}$ with $\alpha(G)=x$ and $\theta(G)=\left\lfloor\frac{8}{5} \alpha(G)\right\rfloor$.

Our result improves on the previous upper bound of $\frac{5}{3} x$ from [4]. The rest of the section is devoted to proving Theorem 2.1. It is best possible because of the graph $G_{5,8}$ represented in Fig. 1 satisfies

$$
\left|V\left(G_{5,8}\right)\right|=15, \omega\left(G_{5,8}\right)=2, \chi\left(G_{5,8}\right)=3, \alpha\left(G_{5,8}\right)=5, \theta\left(G_{5,8}\right)=8 \text {. }
$$


Other graphs with the same parameters can also be found as induced subgraphs in some of the seven graphs with parameters $|V(G)|=17, \omega(G)=2$, $\alpha(G)=5$ (one is given in [7], all seven in [6]). However, $G_{5,8}$ is much simpler for our purposes. Checking $\left|V\left(G_{5,8}\right)\right|=15, \chi\left(G_{5,8}\right)=3$ and $\theta\left(G_{5,8}\right) \leq 8$ is immediate from the figure (cycles of length 5 are easy to find, a 3-colouring is shown and a clique cover of size 8 is obtained by taking every second edge on the obvious hamiltonian cycle and an isolated vertex). Note that $\chi\left(G_{5,8}\right)=3$ implies that $G \in \mathcal{C}$. To compute $\alpha$ and $\omega$, it is convenient to consider the graph $R_{3,5}$, also represented in Fig. 1, that is well known in Ramsey Theory as the unique graph $G$ on at least 13 vertices such that $\omega(G)=2$ and $\alpha(G)=4$. Interestingly, $R_{3,5}$ is also the smallest graph $G$ such that $\theta(G)-\alpha(G) \geq 3$ (see [5]), but we do not use this fact here. Observe that $G_{5,8}$ is obtained from $R_{3,5}$ by subdividing one edge twice, so that $\omega\left(G_{5,8}\right)=2$, and $\theta(G) \geq\left\lceil\left|V\left(G_{5,8}\right)\right| / \omega\left(G_{5,8}\right)\right\rceil=8$. A colour class in $G_{5,8}$ is a stable set of size 5 , and it is easy to check that a stable set of size at least 6 in $G_{5,8}$ would contain a stable set of size 5 of $R_{3,5}$, a contradiction, so $\alpha\left(G_{5,8}\right)=5$.

We now show how to construct a graph $G$ with $\alpha(G)=x$ and $\theta(G)=$ $\left\lfloor\frac{8}{5} \alpha(G)\right\rfloor$ for each integer $x \geq 0$. Define a graph $G$ consisting of $k=\left\lfloor\frac{x}{5}\right\rfloor$ disjoint copies of $G_{5,8}$. Thus, $\theta(G)=8 k, \alpha(G)=5 k$. If $x$ is a multiple of 5 , then $\theta(G)=\left\lfloor\frac{8}{5} \alpha(G)\right\rfloor$ since $\alpha(G)=x$ and $\theta(G)=\frac{8}{5} x$. If $x \equiv 1$ mod 5 , then add an isolated vertex to $G$, so that $x=\alpha(G)=5 k+1$ and $\theta(G)=8 k+1=\left\lfloor\frac{8 x}{5}\right\rfloor$. If $x \equiv 2 \bmod 5$, then add to $G$ a pentagon, so that $x=\alpha(G)=5 k+2$ and $\theta(G)=8 k+3=\left\lfloor\frac{8 x}{5}\right\rfloor$. If $x \equiv 3 \bmod 5$, then add to $G$ a pentagon and an isolated vertex, so that $x=\alpha(G)=5 k+3$ and $\theta(G)=8 k+4=\left\lfloor\frac{8 x}{5}\right\rfloor$. If $x \equiv 4 \bmod 5$, then add to $G$ two pentagons, so that $x=\alpha(G)=5 k+4$ and $\theta(G)=8 k+6=\left\lfloor\frac{8 x}{5}\right\rfloor$.

To prove the upper bound on $\theta$, we need the next results. The following avoids checking small cases.

Lemma 2.2 (Gyárfás, Sebö and Trotignon [5]). If $G$ is a graph on at most 9 vertices, then $\theta(G)-\alpha(G) \leq 1$. If $G$ is a graph on at most 12 vertices, then $\theta(G)-\alpha(G) \leq 2$.

A graph $G$ is $\theta$-critical if for every vertex $v$ of $G, \theta(G-v)<\theta(G)$. The following is a basic result of Gallai (a short proof can be found in [13]).

Theorem 2.3 (Gallai [3]). If $G$ is connected and $\theta$-critical, then

$$
\theta(G) \leq \frac{|V(G)|+1}{2}
$$


It remains to prove that every graph $G$ in $\mathcal{C}$ satisfies $\theta(G) \leq \frac{8}{5} \alpha(G)$. We prove this by induction on $|V(G)|$. For graphs on at most one vertex, the outcome clearly holds. If $G$ is not $\theta$-critical, then for some vertex $v$, by the induction hypothesis, we have

$$
\theta(G)=\theta(G-v) \leq \frac{8}{5} \alpha(G-v) \leq \frac{8}{5} \alpha(G)
$$

If $G$ is disconnected, then $G$ is the disjoint union of two non-empty graphs $H_{1}$ and $H_{2}$, so by the induction hypothesis

$$
\theta(G)=\theta\left(H_{1}\right)+\theta\left(H_{2}\right) \leq \frac{8}{5}\left(\alpha\left(H_{1}\right)+\alpha\left(H_{2}\right)\right)=\frac{8}{5} \alpha(G) .
$$

Thus, we may assume that $G$ is $\theta$-critical and connected. By Theorem 2.3,

$$
\theta(G) \leq \frac{|V(G)|+1}{2}
$$

If $\alpha(G) \geq 5$, then

$$
\theta(G) \leq \frac{|V(G)|+1}{2} \leq \frac{3 \alpha(G)+1}{2} \leq \frac{3 \alpha(G)+1}{2}+\frac{\alpha(G)-5}{10}=\frac{8}{5} \alpha(G) .
$$

If $\alpha(G)=4$, then $|V(G)| \leq 12$, so by Lemma $2.2, \theta(G) \leq \alpha(G)+2=6$, and $\theta(G) \leq 6<8 \times 4 / 5=\frac{8}{5} \alpha(G)$ is clear. If $\alpha(G)=3$, then $|V(G)| \leq 9$, so by Lemma $2.2, \theta(G) \leq \alpha(G)+1=4$, and $\theta(G) \leq 4<8 \times 3 / 5=\frac{8}{5} \alpha(G)$ is clear. If $\alpha(G)=2$, then $|V(G)| \leq 6$, so by Lemma $2.2, \theta(G) \leq \alpha(G)+1=3$, and $\theta(G) \leq 3<8 \times 2 / 5=\frac{8}{5} \alpha(G)$ is clear. If $\alpha(G) \leq 1$, then $G$ is a clique, so the outcome holds.

\section{A complementary bounding function for eventually identity functions}

Let $F_{c}$ denote the class of those $\mathbb{N} \rightarrow \mathbb{N}$ functions such that $f(x)=x$ for $x \geq c$. The following was stated without proof in [4].

Theorem 3.1. For all $c$ and $g \in F_{c}, g$ is complementary-bounded.

Proof. We prove by induction on $c$. For $c=1$ only $g(x)=x$ is in $F_{c}$ and the Perfect Graph Theorem [10] implies that $g(x)=x$ is a complementary bounding function.

Suppose that for some $c \geq 1$ every $f \in F_{c}$ has a complementary bounding function $f_{c}^{*}$ and let $G$ be a graph with $\chi$-bounding function $g \in F_{c+1}$. 
Consider a subgraph $H \subseteq G$ with $\alpha(H)=k$. Let $S=\left\{s_{1}, s_{2}, \ldots, s_{k}\right\}$ be a stable set in $H$. Partition $V(H) \backslash S$ into $A_{1}=N\left(s_{1}\right)$ and for $i=2, \ldots, k$, $A_{i}=N\left(s_{i}\right) \backslash\left(\cup_{j=1}^{i-1} A_{j}\right)$.

We claim that for $1 \leq i \leq k$, each $A_{i}$ induces a graph $H_{i} \subset H$ such that $H_{i}$ has a $\chi$-bounding function in $F_{c}$. Indeed, if $H_{i}$ has a subgraph $G_{i}$ with $p=\omega\left(G_{i}\right)<\chi\left(G_{i}\right)$ for $p>c$, then $c+1<p+1=\omega\left(G_{i} \cup s_{i}\right)<\chi\left(G_{i} \cup s_{i}\right)$ contradicting the assumption that $G$ is $\chi$-bounded by $g$. Thus, the claim is true, so using $\alpha\left(H_{i}\right) \leq k-i+1$ and the induction hypothesis, $\theta\left(H_{i} \cup s_{i}\right)=$ $\theta\left(H_{i}\right) \leq f_{c}^{*}(k-i+1), G$ has a clique cover with $\sum_{j=1}^{k} f_{c}^{*}(k-j+1)$ cliques. Thus, $\sum_{j=1}^{k} f_{c}^{*}(k-j+1)$ is a complementary bounding function for $g$.

For $c=2$, this proof provides the following.

Theorem 3.2. $f^{*}(x)=\left(\begin{array}{c}x+1 \\ 2\end{array}\right)$ is a complementary bounding function for any $f \in F_{2}$.

In fact, the bound provided by Theorem 3.2 is at most a logarithmic factor apart from best possible, since there are triangle-free graphs $G$ with at least $\frac{c \alpha(G)^{2}}{\log \alpha(G)}$ vertices, see [8]. For such $G, \theta(G) \geq \frac{c \alpha(G)^{2}}{2 \log \alpha(G)}$.

Theorem 3.1 invites another question, that of finding $f^{*}$ for $f \in F_{t}$ (with better bounds than Theorem 3.1). The first case beyond the Perfect Graph Theorem (Problem 6.6 in [4]) is not even known.

Problem 3.3. Find $g^{*}$ for the almost identity function

$$
g(x)= \begin{cases}3 & \text { for } x=2 \\ x & \text { for } x>2\end{cases}
$$

From Theorem 3.2, $g^{*}(x) \leq\left(\begin{array}{c}x+1 \\ 2\end{array}\right)$ but it is possible that $g^{*}$ is linear. In ([4] p. 439), the conjecture 'perhaps $g^{*}(x)=\left\lfloor\frac{3 x}{2}\right\rfloor$ is the truth' was raised, based on the example of disjoint circuits of length 5 . This is in fact disproved by the graph $G_{5,8}$ represented in Fig. 1 . From Theorem 3.2 and $G_{5,8}$ we have $g^{*}(2)=3$. While $g^{*}(3) \geq 4$ is obvious (from the pentagon and an isolated vertex), it is not clear whether $g^{*}(3)=4$. Theorem 3.2 suggests the following.

Conjecture 3.4. $g^{*}(x)=\left\lfloor\frac{8}{5} x\right\rfloor$.

\section{Functions that are not complementary-bounded}

In this section, we show that $f(x)=x+x / \operatorname{polylog}(x)$ is not complementarybounded. We prove this by exhibiting a class of graphs $\chi$-bounded by $f$ but not $\theta$-bounded. This family consists of Schrijver graphs which we define 
below (in fact, for convenience we work in the complement, so our graphs will be $\theta$-bounded and not $\chi$-bounded, but this is clearly equivalent up to a complementation).

We provide in Lemma 4.2 a tool to determine a $\theta$-bounding function of any graph with "high" stability ratio (ratio between the stability number and number of vertices) and such that this property is closed under taking induced subgraphs. It relies on the following theorem due to Gallai. We denote by $\nu(G)$ the size of a maximum matching in $G$.

Theorem 4.1 (Gallai [2]). If $G$ is a connected graph such that for all vertices $v, \nu(G \backslash v)=\nu(G)$, then $G$ is factor-critical.

Lemma 4.2. For every graph $G$

$$
\theta(G) \leq \alpha(G)+\max _{H \subseteq G}(|V(H)|-2 \alpha(H))
$$

Proof. We prove the result by induction on $|V(G)|$. It clearly holds when $|V(G)| \leq 1$. Note that $\max _{H \subseteq G}(|V(H)|-2 \alpha(H)) \geq 0$ as we can choose $H$ to be the empty graph.

Case 1. $G$ contains a triangle $T$.

$$
\begin{aligned}
\theta(G) & \leq 1+\theta(G \backslash T) \\
& \leq 1+\alpha(G \backslash T)+\max _{H \subseteq G \backslash T}(|V(H)|-2 \alpha(H)) \\
& \leq \alpha(G)+\left(1+\max _{H \subseteq G \backslash T}(|V(H)|-2 \alpha(H))\right) \\
& \leq \alpha(G)+\max _{H \subseteq G}(|V(H)|-2 \alpha(H)) .
\end{aligned}
$$

Case 2. There exists a vertex $v \in V(G)$ such that $\theta(G \backslash v)=\theta(G)$. By the induction hypothesis,

$$
\begin{aligned}
\theta(G) & =\theta(G \backslash v) \leq \alpha(G \backslash v)+\max _{H \subseteq G \backslash v}(|V(H)|-2 \alpha(H)) \\
& \leq \alpha(G)+\max _{H \subseteq G}(|V(H)|-2 \alpha(H)) .
\end{aligned}
$$

Case 3. $G$ is triangle-free and for every vertex $v \in V(G), \theta(G \backslash v)<\theta(G)$. We suppose that $G$ is connected for otherwise, we obtain the result by the induction hypothesis on the connected components of $G$. Observe that for every vertex $v \in V(G), \theta(G \backslash v)=\theta(G)-1$. Since $G$ is triangle-free,

$$
\theta(G)+\nu(G)=|V(G)| \text { and } \quad \theta(G \backslash v)+\nu(G \backslash v)=|V(G \backslash v)| .
$$


Thus, for every vertex $v \in V(G), \nu(G \backslash v)=\nu(G)$. By Theorem 4.1, $G$ is factor-critical and thus, $\theta(G)=(|V(G)|+1) / 2$.

Also, if $G$ is bipartite, then the result holds by Theorem 1.1. Thus, from here on, we suppose that $G$ is not bipartite. An odd cycle $H$ of minimum length in $G$ is chordless, because a chord would allow us to construct a smaller odd cycle. It follows that $\max _{H \subseteq G}(|V(H)|-2 \alpha(H)) \geq 1$. Now,

$$
\begin{aligned}
\theta(G) & =\frac{|V(G)|+1}{2} \\
& \leq \frac{2 \alpha(G)+\max _{H \subseteq G}(|V(H)|-2 \alpha(H))+1}{2} \\
& =\alpha(G)+\max _{H \subseteq G}(|V(H)|-2 \alpha(H))+\frac{-\max _{H \subseteq G}(|V(H)|-2 \alpha(H))+1}{2} \\
& \leq \alpha(G)+\max _{H \subseteq G}(|V(H)|-2 \alpha(H)) .
\end{aligned}
$$

We now describe subgraphs of Kneser graphs that are not $\chi$-bounded. When $n, k$ are integers, the Kneser graph $K G_{n, k}$ is the graph whose vertices are the subsets of $\{1,2, \ldots, 2 n+k\}$ that have size $n$, and such that two vertices are adjacent if they are disjoint sets. We need several properties of Kneser graphs.

Lemma 4.3. If $H$ is an induced subgraph of $K G_{n, k}$, then

$$
\alpha(H) \geq \frac{n}{2 n+k}|V(H)|
$$

Proof. Let $N=\mid\{(i, v): v \in V(H)$ and $i \in v\}|=n| V(H) \mid$. Suppose that each integer of $\{1, \ldots, 2 n+k\}$ is contained in less than $(n / 2 n+k)|V(H)|$ vertices of $H$. Then $N=\sum_{1 \leq i \leq 2 n+k}|\{v: i \in v\}|<(2 n+k) n /(2 n+$ $k)|V(H)|=n|V(H)|=N$, a contradiction. Therefore, at least one integer of $\{1, \ldots, 2 n+k\}$ is contained in at least $\frac{n}{2 n+k}|V(H)|$ vertices of $H$, that form a stable set of $H$.

Lemma 4.4. If $G$ is an induced subgraph of $K G_{n, k}$ then

$$
\theta(G) \leq\left(1+\frac{k}{n}\right) \alpha(G)
$$

Proof. By Lemma 4.2,

$$
\theta(G) \leq \alpha(G)+\max _{H \subseteq G}(|V(H)|-2 \alpha(H))
$$


Since by Lemma $4.3,|V(H)| \leq\left(2+\frac{k}{n}\right) \alpha(H)$, we have:

$$
\theta(G) \leq \alpha(G)+\max _{H \subseteq G} \frac{k}{n} \alpha(H) \leq \alpha(G)+\frac{k}{n} \alpha(G) .
$$

An $n$-element subset $S$ of $\{1, \ldots, 2 n+k\}$ is sparse if it does not contain two consecutive numbers in the circular ordering of $\{1, \ldots, 2 n+k\}$. The Schrijver graph $S G_{n, k}$ is the subgraph of $K G_{n, k}$ induced by the sparse sets.

Lemma 4.5. $\left|V\left(S G_{n, k}\right)\right|=\left(\begin{array}{c}n+k \\ n\end{array}\right)+\left(\begin{array}{c}n+k-1 \\ n-1\end{array}\right)=\left(\begin{array}{c}n+k \\ k\end{array}\right)+\left(\begin{array}{c}n+k-1 \\ k\end{array}\right)$.

Proof. By increasing $n$-tuple $\{1, \ldots, a\}$, we mean an $n$-tuple $\left(i_{1}, \ldots, i_{n}\right)$ such that for all $1 \leq j \leq n$, we have $1 \leq i_{j} \leq a$ and such that $i_{1}<\cdots<i_{n}$.

Let us first count the sparse subsets of $\{1, \ldots, 2 n+k\}$ that do not contain $2 n+k$. They are in one-to-one correspondence with the increasing $n$-tuples of $\{1, \ldots, n+k\}$. This is clear by considering the map $\left(i_{1}, \ldots, i_{n}\right) \mapsto\left(i_{1}, i_{2}+\right.$ $\left.1, \ldots, i_{n}+n-1\right)$. Therefore, there are $\left(\begin{array}{c}n+k \\ n\end{array}\right)$ sparse subsets of $\{1, \ldots, 2 n+k\}$ that do not contain $2 n+k$.

Let us now count the sparse subsets of $\{1, \ldots, 2 n+k\}$ that do contain $2 n+k$. They are in one-to-one correspondence with the increasing $(n-1)$-tuples of $\{1, \ldots, n+k-1\}$. This is clear by considering the map $\left(i_{1}, \ldots, i_{n-1}\right) \mapsto\left(i_{1}+1, i_{2}+2, \ldots, i_{n-1}+n-1,2 n+k\right)$. Therefore, there are $\left(\begin{array}{c}n+k-1 \\ n-1\end{array}\right)$ sparse subsets of $\{1, \ldots, 2 n+k\}$ that do contain $2 n+k$.

The conclusions of the two paragraphs above sum up to the first equality, and the second follows from $\left(\begin{array}{c}a+b \\ a\end{array}\right)=\left(\begin{array}{c}a+b \\ b\end{array}\right)$.

The following is the key property of Kneser and Schrijver graphs.

Theorem 4.6 (Lovász [11], Schrijver [12]) $\chi\left(K G_{n, k}\right)=\chi\left(S G_{n, k}\right)=k+2$.

We are now ready to exhibit functions that are not complementarybounded.

Theorem 4.7. Let $h$ be a non-decreasing function such that for all $k$, $h\left(n^{k}\right) \leq n / k$ for sufficiently large $n$. Then $x+x / h(x)$ is not complementarybounded.

Proof. It is easy to show that for all $k, h\left(2(n+k)^{k}\right) \leq \frac{n}{k}$ for sufficiently large $n$ (say for $n \geq N(k)$ ). We can show this by simply choosing the $N(k)=$ $N^{\prime}(2 k+1)$ where $N^{\prime}$ is the threshold needed for $h\left(N^{k}\right) \leq N / k$ and using the monotonicity of $h$. Now, for all $n \geq N(k), h\left(2(n+k)^{k}\right) \leq h\left(n(n+n)^{k}\right)=$ $h\left(n^{2 k+1}\right) \leq n / k$.

By Lemma 4.5, for any $k, n$ and any subgraph $H$ of $S G_{2 n+k, n}$, we have

$$
\alpha(H) \leq|V(H)| \leq\left|V\left(S G_{2 n+k, n}\right)\right|=\left(\begin{array}{c}
n+k \\
k
\end{array}\right)+\left(\begin{array}{c}
n+k-1 \\
k
\end{array}\right) \leq 2(n+k)^{k} .
$$


We claim the graphs $\mathcal{S}=\left\{\mathcal{S G}_{\in \mathcal{N}(\|)+\|} \mid \| \in \mathbb{N}\right\}$ are $\theta$-bounded by $f$ (they are not $\chi$-bounded as they are Schrijver graphs).

Since $h$ is non-decreasing, for a subgraph $H$ of $S G_{2 n+k} \in \mathcal{S}$,

$$
\frac{1}{h(\alpha(H))} \geq \frac{1}{h\left(2(n+k)^{k}\right)} \geq \frac{k}{n} .
$$

Thus,

$$
f(\alpha(H))=\alpha(H)\left(1+\frac{1}{h(\alpha(H))}\right) \geq \alpha(H)\left(1+\frac{k}{n}\right) \geq \theta(H)
$$

by Lemma 4.3 . Thus, $\mathcal{S}$ is $\theta$-bounded by $f$ but not $\chi$-bounded, as required.

Theorem 4.8. The function $f(x)=x+x / \log ^{j}(x)$ is not complementarybounded for any $j \in \mathbb{R}$.

Proof. We only need to verify that for all $k, \log ^{j}\left(n^{k}\right) \leq n / k$ for sufficiently large $n$ (and apply Theorem 4.7).

Given $k$, choose $N$ large enough so $N \geq \log ^{2 j+1}(N)$ and $\log (N) \geq k$. Then for any $n \geq N$,

$$
k \log ^{j}\left(n^{k}\right)=k^{j+1} \log ^{j}(n) \leq \log ^{j+1}(n) \log ^{j}(n)=\log ^{2 j+1}(n) \leq n
$$

as required.

\section{References}

[1] P. Erdős (1974). Some new applications of probability methods to combinatorial analysis and graph theory. Proc. 5-th Southeastern Conf. on Combinatorics, Graph Theory and Computing, Congressus Numerantium 10 39-51. MR0364020

[2] T. Gallai (1963). Neuer Beweis eines Tutte'schen Satzes. A Magyar Tudományos Akadémia - Matematikai Kutató Intézetének Közleményei 8 135-139. MR0166777

[3] T. Gallai (1963). Kritische Graphen II. A Magyar Tudományos Akadémia - Matematikai Kutató Intézetének Közleményei 8 373-395. MR0188100

[4] A. Gyárfás (1987). Problems from the world surrounding perfect graphs. Zastowania Matematyki Applicationes Mathematicae 19 413441. MR0951359 
[5] A. Gyárfás, A. Sebő, and N. Trotignon (2012). The chromatic gap and its extremes. Journal of Combinatorial Theory, Series B 102 11551178. MR2959396

[6] J. G. Kalbfleisch (1965). Construction of special edge-chromatic graphs. Canadian Mathematical Bulletin 8 575-584. MR0193026

[7] G. Kéri (1964). On a theorem of Ramsey. Matematikai Lapok $15204-$ 224. In Hungarian. MR0172264

[8] J. H. Kim (1995). The Ramsey number $R(3, t)$ has order of magnitude $t^{2} / \log$ t. Random Structures and Algorithms 7(3) 173-208. MR1369063

[9] D. König (1931). Graphok és Matrixok. Matematikai és Fizikai Lapok 38 116-119.

[10] L. Lovász (1972). Normal hypergraphs and the perfect graph conjecture. Discrete Mathematics 2 253-267. MR0302480

[11] L. Lovász (1978). Kneser's conjecture, chromatic number, and homotopy. Journal of Combinatorial Theory, Series A 25(3) 319-324. MR0514625

[12] A. Schrijver (1978). Vertex-critical subgraphs of Kneser graphs. Nieuw Archief voor Wiskunde 3(XXVI) 454-461. MR0512648

[13] M. Stehlík (2003). Critical graphs with connected complements. Journal of Combinatorial Theory, Series B 89(2) 189-194. MR2017723

ANDRÁs GyÁRFÁs

Alfréd RÉnyi Institute of Mathematics

Hungarian ACADEMy OF SCIENCES

Budapest, P.O. Box 127, H-1364

HUNGARY

E-mail address: gyarfas.andras@renyi.mta.hu

Zhentao Li

École Normale Supérieure de Lyon, LIP, Équipe MC2

46, Allée D’Italie, 69342 Lyon Cedex 07

FRANCE

E-mail address: zhentao.li@ens-lyon.fr

RAPHAEL MACHADO

INMETRo, Rio DE JANEIRO

BRAZIL

E-mail address: rcmachado@inmetro.gov.br 
ANDRÁS SEBŐ

CNRS, Grenoble-INP, UJF, Laboratoire G-SCOP

EQUipe Optimisation COMBINATOIRE

46 avenue Félix Viallet, 38031 Grenoble Cedex

FRANCE

E-mail address: andras.sebo@g-scop.inpg.fr

STÉPHAN THOMAsSÉ

École Normale Supérieure de Lyon, LIP, Équipe MC2 46, allée D'Italie, 69342 Lyon Cedex 07

FRANCE

E-mail address: stephan.thomasse@ens-lyon.fr

Nicolas TRotignon

CNRS, École Normale Supérieure de Lyon, LIP, UCBL

Université de Lyon, INRIA, ÉQUIPE MC2

46, allée D‘Italie, 69342 Lyon Cedex 07

FRANCE

E-mail address: nicolas.trotignon@ens-lyon.fr

ReCEIVEd APRIL 9, 2013 